\title{
Introducing localized constraints in global geomagnetic field modelling
}

\author{
Vincent Lesur \\ British Geological Survey, Murchison House, West Mains Road, Edinburgh, EH9 3LA, UK
}

(Received November 18, 2004; Revised August 16, 2005; Accepted August 25, 2005; Online published April 14, 2006)

\begin{abstract}
A set of functions is defined that can be used for modelling the internal part of the geomagnetic field. These functions are represented in term of spherical harmonics of a given maximum degree $L$ and are centred at specific latitudes and longitudes. The number of functions needed and the positions of their centres are such that any potential field of maximum spherical harmonic degree $L$ can be modelled. Formulae are obtained to transform between the potential field representation using these functions and a classic spherical harmonic representation. The shape of these functions can be optimized to make them reasonably localized, and from there it is shown how a localized constraint can be applied to an internal geomagnetic field model. The technique is demonstrated by means of models built from a few months of the Swarm mission synthetic data set.
\end{abstract}

Key words: Geomagnetism, Geomagnetic Field, Swarm, Localized constraints, wavelets.

\section{Introduction}

One of the major difficulties when building a geomagnetic field model is to estimate the weights to be given to the different data sets and, in the data sets themselves, the appropriate weight for each data value. The data with uncorrelated errors should be weighted by the inverse of their variances but usually these variances are not known. Furthermore, geomagnetic field models do not generally model all the sources of magnetic field and the data weights should take into account the part of the signal that is not modelled. Again, estimating accurately the amplitude of these signals is very difficult, if not impossible.

The usual approach when building spherical harmonic models of the geomagnetic field from satellite data is to weight the data to take into account the higher data density close to the poles and the increase in noise level at high latitude. This approach, however, is not always satisfactory. The problem would not be so acute if, in place of a spherical harmonic representation, local basis functions were used. In this case, localized noisy data would not have any influence on a local basis function parameter situated far away and, if necessary, a constraint could be applied locally to control the field model behaviour wherever there are noisy, sparse or poor quality data.

Local basis functions can be seen as a special case of wavelets and theoretical work has been done to develop wavelets on a sphere (Freeden et al., 1998). There are very few models of the internal geomagnetic field built using wavelets. Maier and Mayer (2003) present an application to the crustal magnetic field. Another example is presented in Holschneider et al. (2003) where the authors used Poisson multipole wavelets that contain all spherical harmonic degrees. In the present work we develop a set of functions for the representation of the internal magnetic field where each function can be described with only spherical harmonics of

(C) NERC, 2006. All Rights Reserved. degrees less than or equal to a given value $L$. These functions are a special case of "band-limited wavelets" (Freeden et al., 1998). We choose to limit the maximum spherical harmonic degree of the functions to have good control on the downward continuation process associated with the use of satellite data. Because our functions do not contain spherical harmonic degrees larger than $L$, we define and place them on the sphere in such a way that any harmonic function of spherical harmonic degree less than or equal to $L$ can be represented. Therefore, we will see that it is easy to transform between a spherical harmonic representation of a potential field and a representation using our set of functions. Indeed, since we have only a finite number of spherical harmonic degrees we can only build "quasi-local" functions that have very small amplitude outside a given latitude and longitude window (but not zero as would be expected for truly local functions). However, we will see that we can make them reasonably localized, and as a result, it is easy to implement localized constraints.

In the next section, the theory is presented, and in the third section an example is given of an application using the Swarm synthetic satellite data set (Olsen et al., 2006).

\section{Theory}

A general description of wavelets on the sphere and, more specifically, band-limited wavelets can be found in Freeden et al. (1998), but in the following we concentrate on the representation we choose. Then, formulae are established to transform a spherical harmonic representation into the new system of representation, and conversely. The functions used for the representation are then optimized to make them as local as possible. Finally localized constraints are introduced.

Let $H a_{L}(\Omega)$ be the space of the restriction of all harmonic functions of maximum degree $L$ to the unit sphere $\Omega$. If $g(\theta, \varphi)$ is a function of $\mathrm{Ha}_{L}(\Omega)$, it can be expressed as a linear combination of Schmidt normalized spherical har- 
monics $Y_{l}^{m}(\theta, \varphi)^{1}$ :

$$
g(\theta, \varphi)=\sum_{l=0}^{L} \sum_{m=-l}^{l} \tilde{g}_{l}^{m} Y_{l}^{m}(\theta, \varphi)
$$

The negative orders $(m<0)$ are associated with $\sin (m \varphi)$ terms, whereas zero or positive orders $(m \geq 0)$ are associated with $\cos (m \varphi)$ terms. The $\tilde{g}_{l}^{m}$ are calculated by integration:

$$
\tilde{g}_{l}^{m}=\frac{2 l+1}{4 \pi} \int_{0}^{2 \pi} \int_{0}^{\pi} g(\theta, \varphi) Y_{l}^{m}(\theta, \varphi) \sin \theta d \theta d \varphi
$$

We want to use an alternative representation for $g(\theta, \varphi)$ :

$$
g(\theta, \varphi)=\sum_{i, j} \tilde{g}_{i j} F_{i j}^{L}(\theta, \varphi) .
$$

The functions $F_{i j}^{L}(\theta, \varphi)$ are centred on a point $\left(\theta_{i}, \varphi_{j}\right)$ of the unit sphere $\Omega$ and are defined by:

$$
\begin{aligned}
F_{i j}^{L}(\theta, \varphi) & =\sum_{l=0}^{L} \sum_{m=-l}^{l} f_{l} Y_{l}^{m}\left(\theta_{i}, \varphi_{j}\right) Y_{l}^{m}(\theta, \varphi) \quad f_{l} \neq 0 \\
& =\sum_{l=0}^{L} f_{l} P_{l}(\cos \mu)
\end{aligned}
$$

where $\mu$ is the angle between the two unit vectors pointing in the directions $\left(\theta_{i}, \varphi_{j}\right)$ and $(\theta, \varphi)$ and $P_{l}(\cos \mu)$ are the Legendre polynomials of degree $l$ (see Backus et al., 1996, p. 141). Obviously the functions $F_{i j}^{L}(\theta, \varphi)$ are in $H a_{L}(\Omega)$. We show below that for any function $g(\theta, \varphi)$ of $H a_{L}(\Omega)$ to be defined in terms of $F_{i j}^{L}(\theta, \varphi)$, as in Eq. (3), only $(L+1)(2 L+1)$ functions $F_{i j}^{L}(\theta, \varphi)$ are needed, centred at the $\left(\theta_{i}, \varphi_{j}\right)$ given by:

$$
\begin{aligned}
\theta_{i} & =\arccos \left(x_{i}\right) \\
\varphi_{j} & =\frac{2 \pi j}{(2 L+1)}
\end{aligned}
$$

where $x_{i}$ is the $i$ th zero of the Legendre polynomial $P_{L+1}(x)$.

This is a direct consequence of the following sampling theorem on $\Omega$ :

Theorem-1: Let $\tilde{g}_{l}^{m}$ be the spherical harmonic coefficients of a function $g(\theta, \varphi)$ of $H a_{L}(\Omega) \quad$ (i.e. $\tilde{g}_{l}^{m}=0$ for $l>L$ ) then:

$$
\tilde{g}_{l}^{m}=\frac{2 l+1}{2(2 L+1)} \sum_{i=1}^{L+1} w_{i}^{L+1} \sum_{j=1}^{2 L+1} g\left(\theta_{i}, \varphi_{j}\right) Y_{l}^{m}\left(\theta_{i}, \varphi_{j}\right)
$$

for $l \leq L$ and $-l \leq m \leq l$. The $\left(\theta_{i}, \varphi_{j}\right)$ are defined as in (5) and the weights $w_{i}^{L+1}$ are given by:

$$
w_{i}^{L+1}=\frac{2}{\left(1-x_{i}^{2}\right)}\left(\partial_{x} P_{L+1}\left(x_{i}\right)\right)^{-2} \quad i=1, \cdots, L+1
$$

We do not give here a proof for this theorem that is often used in geophysics (e.g., Lesur and Gubbins, 1999 or

${ }^{1}$ In our formulation we will include the spherical harmonic of zero degree and order, but it can be excluded for geomagnetic applications.
Sneeuw, 1994). An alternative sampling theorem could be used with regular sampling both in $\theta$ and $\varphi$ (Driscoll and Healy, 1994) but twice as many functions $F_{i j}^{L}(\theta, \varphi)$ would then be required. To the author's knowledge an exact sampling theorem on the sphere requiring less than $(L+1)(2 L+1)$ sampling points has not been established yet. By using such a theorem, one would be able to reproduce the results presented below with fewer functions $F_{i j}^{L}(\theta, \varphi)$.

We can rewrite Eq. (6) for the function $Y_{l^{\prime}}^{m^{\prime}}(\theta, \varphi), l^{\prime} \leq L$ :

$$
\delta_{l l^{\prime}} \delta_{m m^{\prime}}=\frac{2 l^{\prime}+1}{2(2 L+1)} \sum_{i=1}^{L+1} \sum_{j=1}^{2 L+1} w_{i}^{L+1} Y_{l^{\prime}}^{m^{\prime}}\left(\theta_{i}, \varphi_{j}\right) Y_{l}^{m}\left(\theta_{i}, \varphi_{j}\right)
$$

where $\delta_{i j}=1$ if $i=j$ and zero otherwise. Starting from Eq. (1) and then introducing Eq. (8) gives:

$$
\begin{aligned}
g(\theta, \varphi)= & \sum_{l, m} \tilde{g}_{l}^{m} Y_{l}^{m}(\theta, \varphi) \\
= & \sum_{l^{\prime}, m^{\prime}} \sum_{l, m} \tilde{g}_{l^{\prime}}^{m^{\prime}} \frac{f_{l}}{f_{l^{\prime}}}\left\{\delta_{l l^{\prime}} \delta_{m m^{\prime}}\right\} Y_{l}^{m}(\theta, \varphi) \\
= & \sum_{l^{\prime}, m^{\prime}} \sum_{l, m} \tilde{g}_{l^{\prime}}^{m^{\prime}} \frac{f_{l}}{f_{l^{\prime}}}\left\{\frac{2 l^{\prime}+1}{2(2 L+1)} \sum_{i=1}^{L+1} \sum_{j=1}^{2 L+1} w_{i}^{L+1}\right. \\
& \left.\cdot Y_{l^{\prime}}^{m^{\prime}}\left(\theta_{i}, \varphi_{j}\right) Y_{l}^{m}\left(\theta_{i}, \varphi_{j}\right)\right\} Y_{l}^{m}(\theta, \varphi) \\
= & \sum_{i=1}^{L+1} \sum_{j=1}^{2 L+1} \sum_{l^{\prime}, m^{\prime}} \frac{\tilde{g}_{l^{\prime}}^{m^{\prime}}}{f_{l^{\prime}}} \frac{2 l^{\prime}+1}{2(2 L+1)} w_{i}^{L+1} \\
& \cdot Y_{l^{\prime}}^{m^{\prime}}\left(\theta_{i}, \varphi_{j}\right)\left\{\sum_{l, m} f_{l} Y_{l}^{m}\left(\theta_{i}, \varphi_{j}\right) Y_{l}^{m}(\theta, \varphi)\right\} \\
= & \sum_{i=1}^{L+1} \sum_{j=1}^{2 L+1} \tilde{g}_{i j} F_{i j}^{L}(\theta, \varphi)
\end{aligned}
$$

where

$$
\tilde{g}_{i j}=\sum_{l^{\prime}=0}^{L} \sum_{m=-l^{\prime}}^{l^{\prime}} \frac{\tilde{g}_{l^{\prime}}^{m^{\prime}}}{f_{l^{\prime}}} \frac{2 l^{\prime}+1}{2(2 L+1)} w_{i}^{L+1} Y_{l^{\prime}}^{m^{\prime}}\left(\theta_{i}, \varphi_{j}\right)
$$

Therefore any function $g(\theta, \varphi)$ defined as in Eq. (1) can be expressed in terms of $F_{i j}^{L}(\theta, \varphi)$, as in Eq. (3), as long as $f_{l} \neq 0$, i.e. the $F_{i j}^{L}(\theta, \varphi)$ span the whole $H a_{L}(\Omega)$ space.

It would be tempting to reduce the required number of functions $F_{i j}^{L}(\theta, \varphi)$ (see for example Cui and Freeden, 1997). However, by doing so, we would not be able to establish the set of Eqs. (9) and Eq. (10). It is because we are using an exact sampling theorem on the sphere that we are able to prove that the $F_{i j}^{L}(\theta, \varphi)$ span the whole $H a_{L}(\Omega)$ space.

The $(L+1)(2 L+1)$ functions $F_{i j}^{L}(\theta, \varphi)$ are not linearly independent since the dimension of $H a_{L}(\Omega)$ is only $(L+1)^{2}$. Therefore there is not a unique decomposition of a function of $\mathrm{Ha}_{L}(\Omega)$ in terms of $F_{i j}^{L}(\theta, \varphi)$. Equation (10) gives a possible set of $\tilde{g}_{i j}$ as a function of the $\tilde{g}_{l}^{m}$. By equating Eqs. (1) and (3), multiplying both sides by $Y_{l}^{m}(\theta, \varphi)$ and integrating over the sphere, it is straightforward to find the 


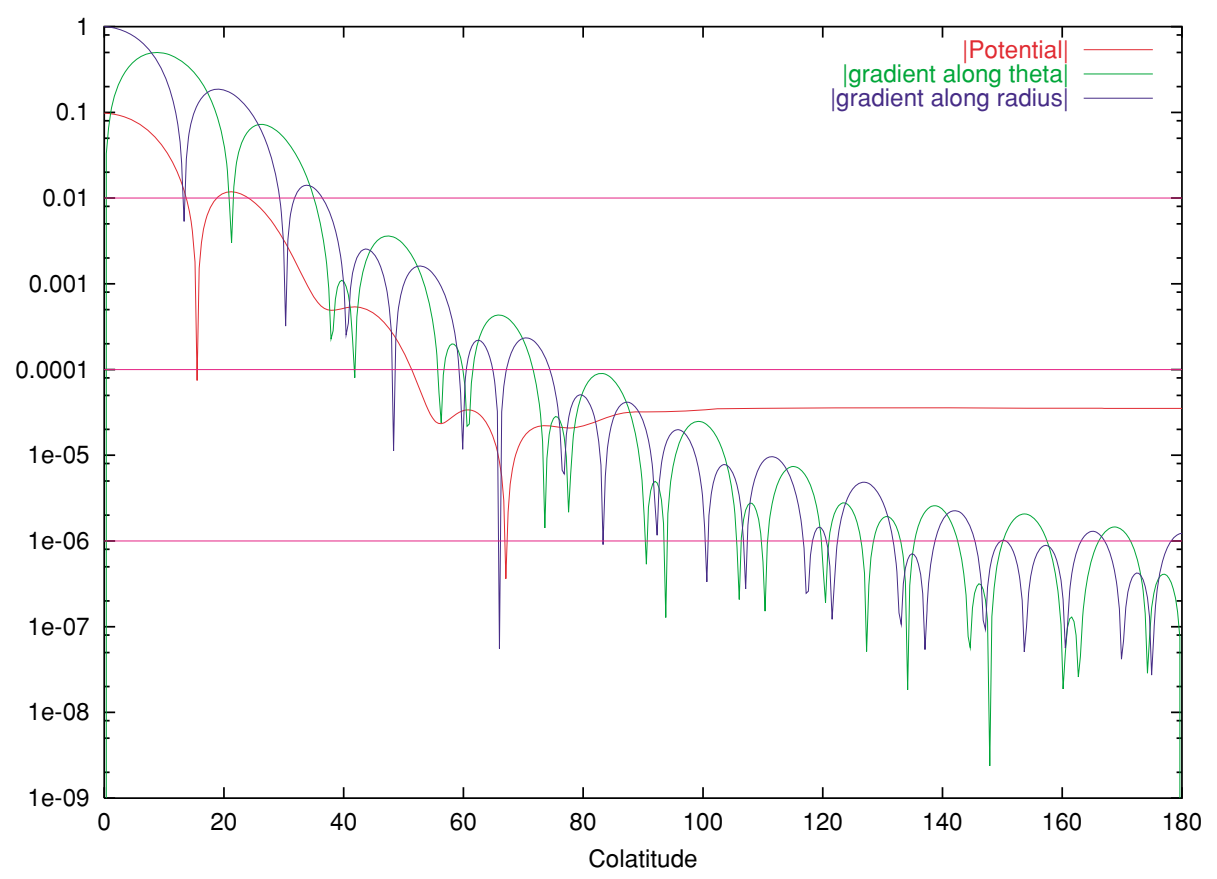

Fig. 1. Normalized absolute value of the potential $F_{00}^{20}(\theta, \varphi, a)$ and its gradients in the $\theta$ and $r$ directions.

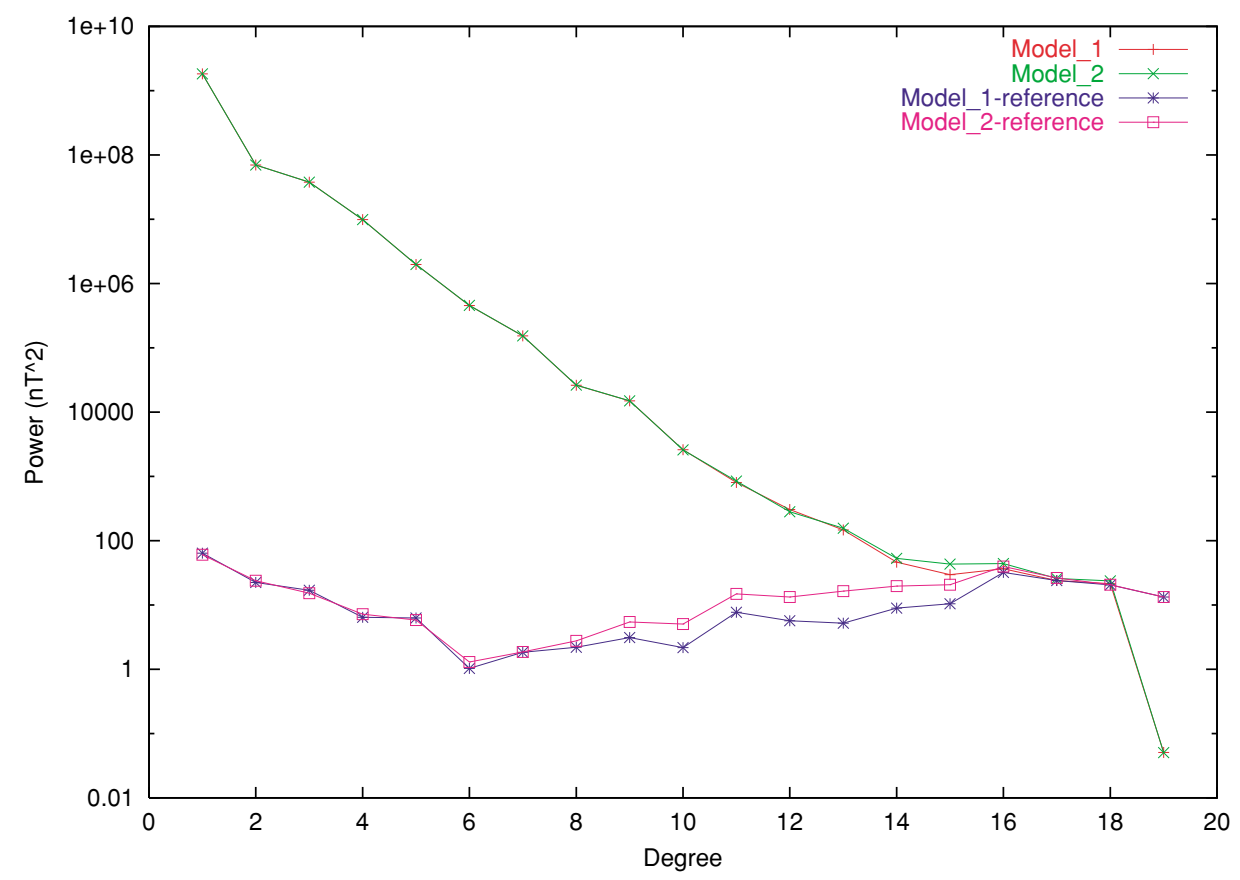

Fig. 2. Power spectra of model_1, model_2 and of their differences relative to the reference field model for 1997.5 .

reciprocal formula:

$$
\tilde{g}_{l^{\prime}}^{m^{\prime}}=\sum_{i=1}^{L+1} \sum_{j=1}^{2 L+1} \tilde{g}_{i j} f_{l^{\prime}} Y_{l^{\prime}}^{m^{\prime}}\left(\theta_{i}, \varphi_{j}\right)
$$

We now consider the problem in the whole 3-D space and define the function $F_{i j}^{L}(\theta, \varphi, r)$ by:

$$
\begin{gathered}
F_{i j}^{L}(\theta, \varphi, r)=a \sum_{l=0}^{L} \sum_{m=-l}^{l}\left(\frac{a}{r}\right)^{l+1} f_{l} Y_{l}^{m}\left(\theta_{i}, \varphi_{j}\right) Y_{l}^{m}(\theta, \varphi) \\
f_{l} \neq 0 r \geq a
\end{gathered}
$$

Finding the best possible $f_{l}$ to build "local" $F_{i j}^{L}(\theta, \varphi, r)$ functions is very similar to the discussion about the " $\delta$ ness" of averaging kernels in Backus and Gilbert (1968) (see also: Backus et al., 1996, p. 153). In the present work, the $f_{l}$ that minimize Eq. (13) below are used, with ad hoc weight function $w_{L}(\theta)$ and associated value $\gamma=0.873$ (in radians). This minimizes the magnitude of the gradient of $F_{00}^{L}(\theta, \varphi, a)$ for all $\theta \neq 0$ (and not the amplitude of $F_{00}^{L}(\theta, \varphi, a)$ because we have ultimately to model a magnetic field that is a gradient of a potential). All the functions $F_{i j}^{L}(\theta, \varphi)$ have the same basic shape and are symmetric rel- 
ative to the vector pointing in the direction of their centre $\left(\theta_{i}, \varphi_{j}\right)$. In Eq. (13), the function $F_{00}^{L}(\theta, \varphi, a)$ is centred on the North Pole so that it does not depend on longitude $\varphi$.

$$
\begin{aligned}
& I=\left\{1-\partial_{r} F_{00}^{L}(0,0, a)\right\}^{2}+\int_{\theta=0}^{\pi}\left\{w_{L}(\theta) \partial_{\theta} F_{00}^{L}(\theta, 0, a)\right\}^{2} d \theta \\
&+\int_{\theta=0}^{\pi}\left\{w_{L}(\theta) \partial_{r} F_{00}^{L}(\theta, 0, a)\right\}^{2} d \theta \\
&\left\{\begin{array}{l}
\theta_{0}=0 \\
\varphi_{0}=0
\end{array} \text { and } w_{L}(\theta)= \begin{cases}\exp \left(-\left(\frac{\theta-\pi}{\gamma}\right)^{2}\right) \\
\text { for } \theta \neq 0\end{cases} \right. \\
&
\end{aligned}
$$

The $f_{l}$ are then normalized such that $\left.\partial_{r} F_{00}^{L}(\theta, 0, a)\right|_{\theta=0}=1$. Figure 1 shows the absolute value of the potential $F_{00}^{20}(\theta, \varphi, a)$ and its gradients $\partial_{\theta} F_{00}^{20}(\theta, 0, a)$ and $\partial_{r} F_{00}^{20}(\theta, 0, a)$ as a function of colatitude $\theta$. These gradients decrease rapidly as $\mu$, the angle between the two unit vectors pointing in the directions $\left(\theta_{0}, 0\right)$ and $(\theta, 0)$, increases. Obviously, if the maximum degree $L$ is increased, functions $F_{00}^{L}(\theta, \varphi, a)$ can be built with gradients that decrease even faster with $\mu$.

In geomagnetic applications, the functions $F_{i j}^{L}(\theta, \varphi, r)$ defined in Eq. (12) can be used to parameterise a magnetic field whose sources are inside a sphere of radius $a$. The magnetic field is a gradient of a potential therefore:

$$
\boldsymbol{B}=-\nabla\left\{\sum_{i=1}^{L+1} \sum_{j=1}^{2 L+1} \tilde{g}_{i j} F_{i j}^{L}(\theta, \varphi, r)\right\}
$$

Finding the $\tilde{g}_{i j}$ that fit a set of geomagnetic data is a problem with no unique solution (because the $F_{i j}^{L}(\theta, \varphi)$ are not linearly independent) and regularization is needed. Further problems can arise if one of the $f_{l}$ is too small, leading to a normal equation matrix that displays (non-zero but) very small eigenvalues. Typically this will happen for large values of " $l$ " when the data acquisition surface is away from the reference surface of radius $a$. For this case, again, some regularization will be needed.

Consider $N(N \leq(L+1)(2 L+1))$ pairs of indices $\{i, j\}$ and the associated subset $S_{N}$ of functions $F_{i j}^{L}(\theta, \varphi, r)$. A magnetic field $\tilde{\boldsymbol{B}}$ is then defined by:

$$
\tilde{\boldsymbol{B}}=-\nabla\left\{\sum_{\{i, j\}} \tilde{g}_{i j} F_{i j}^{L}(\theta, \varphi, r)\right\}
$$

where the $\tilde{g}_{i j}$ are the parameters that define the magnetic field $\boldsymbol{B}$ in Eq. (14). If a constraint is applied for all latitudes and longitudes on $\tilde{\boldsymbol{B}}$, it will only have a local effect on $\boldsymbol{B}$ as long as the $N$ functions $F_{i j}^{L}(\theta, \varphi, r)$ all have their centres $\left(\theta_{i}, \varphi_{j}\right)$ in a restricted latitude and longitude window. In the next section, an internal magnetic field model will be estimated from a data set and a measure of the roughness of its vertical component on the sphere of radius $a$ will be minimized at high latitude. Using the definition of the functions $F_{i j}^{L}(\theta, \varphi, r)$ in Eq. (12), the vertical component of $\tilde{\boldsymbol{B}}$ on the sphere of radius $a$ is:

$$
\tilde{\boldsymbol{B}}_{z}(\theta, \varphi, a)=\sum_{\{i, j\}} \tilde{g}_{i j} \sum_{l, m}(l+1) f_{l} Y_{l}^{m}\left(\theta_{i}, \varphi_{j}\right) Y_{l}^{m}(\theta, \varphi)
$$

The measure of the roughness (i.e. the measure of the amplitude of the second tangential derivative) of the magnetic field $\tilde{\boldsymbol{B}}$ vertical component on the sphere of radius $a$ is then:

$$
\begin{aligned}
I_{a}= & \int_{\Omega_{a}}\left(\nabla_{s}^{2} \tilde{B}_{z}\right)^{2} d \Omega=\sum_{\{i, j\}} \sum_{\{t, s\}} \tilde{g}_{i j} \tilde{g}_{t s} \\
& \cdot \sum_{l=0}^{L} \frac{4 \pi a^{2}}{2 l+1} l^{2}(l+1)^{4} f_{l}^{2} P_{l}(\cos \mu)
\end{aligned}
$$

where $\mu$ is the angle between the two directions $\left(\theta_{i}, \varphi_{j}\right)$ and $\left(\theta_{t}, \varphi_{s}\right)$. We have used the Schmidt normalization relation:

$$
\int_{\Omega_{a}} Y_{l}^{m}(\theta, \varphi) Y_{l^{\prime}}^{m^{\prime}}(\theta, \varphi) d \Omega=\frac{4 \pi a^{2}}{2 l+1} \delta_{l l^{\prime}} \delta_{m m^{\prime}}
$$

and

$$
\nabla_{s}^{2} Y_{l}^{m}(\theta, \varphi)=-l(l+1) Y_{l}^{m}(\theta, \varphi) .
$$

The model of the main magnetic field that minimizes the integral (17) is built by introducing in the least-squares scheme a damping matrix $\boldsymbol{D}$ whose elements associated with the parameters $\tilde{g}_{i j}$ and $\tilde{g}_{t s}$ are given by:

$$
D_{i j, t s}=\sum_{l=0}^{L} \frac{l^{2}(l+1)^{4}}{2 l+1} f_{l}^{2} P_{l}(\cos \mu)
$$

In our approach, a geomagnetic field model is defined by Eq. (14) and the damping matrix defined in (20) is used. Alternatively, the field can be modelled by a spherical harmonic representation and the damping matrix is then the same as above but right multiplied by the matrix defined in Eq. (10) and left multiplied by its transpose. In this latter approach, which we would use for example to build a model of the crustal magnetic field to a high spherical harmonic degree, the advantage is a smaller number of parameters. Building the damping matrix would then require more work but it is straightforward.

\section{Application to a Synthetic Data Set}

In this section we use the Swarm synthetic data set (Olsen et al., 2006) spanning several months in 1997 and compare models with and without local constraints. These models are also compared with the Swarm reference model for 1997.5.

The Swarm synthetic data set was built using the Comprehensive Model (Sabaka et al., 2004), and includes contributions from the core field, the crustal magnetization, the ionospheric and magnetospheric systems of currents (and the fields they induce in the Earth), field-aligned currents (whose associated fields are not necessarily potential fields), and, finally, instrumental noise. We selected data from four satellites (satellites A, B, C and D in Olsen et al., 2006) between 1997.315 and 1997.5479 filtered according to: local-times 23:00-06:00; $K p<1+$ (and $<2-$ in the previous three hours); $-10<D s t<+10$ ( \pm 15 for the previous hour); interplanetary magnetic field data with $-1<B_{z}<+5,\left|B_{y}\right|<3,\left|B_{x}\right|<10 \mathrm{nT}$ and solar wind speed $V s w<450 \mathrm{~km} / \mathrm{s}$. Vector data only were selected in the magnetic latitude range $\left[-60^{\circ}: 60^{\circ}\right]$; elsewhere, scalar data were used. 
Table 1. Mean and $r m s$ misfit to the data set for the two models.

\begin{tabular}{ccccccc}
\hline \multirow{2}{*}{ Component } & Number of & \multicolumn{2}{c}{ No damping $\lambda=0$} & & \multicolumn{2}{c}{ Slight damping $\lambda=5 \times 10^{-6}$} \\
\cline { 6 - 7 } & data & mean & rms & & mean & rms \\
\hline$X$ & 27315 & 0.16 & 8.77 & & 0.23 & 8.75 \\
$Y$ & 27315 & -3.31 & 9.60 & & -3.31 & 9.59 \\
$Z$ & 27315 & 0.31 & 5.81 & & 0.42 & 5.94 \\
$F$ & 15017 & 5.19 & 7.65 & & 6.41 & 10.40 \\
\hline
\end{tabular}

These data were fitted with a very simple geomagnetic field model:

$$
\begin{aligned}
\boldsymbol{B}= & -\nabla\left\{\sum_{i=1}^{L+1} \sum_{j=1}^{2 L+1} \tilde{g}_{i j} F_{i j}^{L}(\theta, \varphi, r)\right. \\
& \left.+a \sum_{l=1}^{2} \sum_{m=-l}^{l} \tilde{q}_{l}^{m}\left(\frac{r}{a}\right)^{l} Y_{l}^{m}(\theta, \varphi)\right\}
\end{aligned}
$$

The model is not time dependent and corresponds roughly to a snapshot model for 1997.5. The functions $F_{i j}^{L}(\theta, \varphi, r)$ are defined in Eq. (12) and the maximum spherical harmonic degree used for the internal part of the model is $L=19$.

This model is defined by 788 parameters (780 internal and 8 external parameters) whereas in the equivalent "spherical harmonic only" representation only 408 parameters (including the $l=0, m=0$ internal parameter) would have been required.

The standard deviations associated with the data are defined by:

$$
\sigma=\sigma_{0}+d_{z}(1+\cos (z a))
$$

where $z a$ is the zenith angle of the sun at $250 \mathrm{~km}$ altitude, the factor $d_{z}=10 \mathrm{nT}$ and $\sigma_{0}=2 \mathrm{nT}$ are used for all data. During the inversion the data are multiplied by weights proportional to the inverse of these standard deviations. To deal with the data density at high latitude, these weights are further divided by the number of data in roughly equal-area cells whose size at the equator was $5^{\circ}$ in latitude and longitude. Since they are selected for the northern hemisphere summer, the data at high (positive) latitudes are sparse, noisy and strongly down-weighted in the inversion process.

The model parameters are estimated using the usual leastsquares iterative process:

$$
\begin{aligned}
\boldsymbol{d}_{i} & =\boldsymbol{G}\left(\boldsymbol{p}_{i}\right) \\
\boldsymbol{\delta} \boldsymbol{d}_{i} & =\boldsymbol{d}_{o b s}-\boldsymbol{d}_{i} \\
\boldsymbol{\delta} \boldsymbol{p}_{i} & =\left[\boldsymbol{G}_{i}^{t} \boldsymbol{W} \boldsymbol{G}_{i}+\lambda \boldsymbol{D}\right]^{-1}\left[\boldsymbol{G}_{i}^{t} \boldsymbol{W} \boldsymbol{\delta} \boldsymbol{d}_{i}-\lambda \boldsymbol{D} \boldsymbol{p}_{i}\right] \\
\boldsymbol{p}_{i+1} & =\boldsymbol{p}_{i}+\boldsymbol{\delta} \boldsymbol{p}_{i}
\end{aligned}
$$

where the superscript ${ }^{i}$ denotes the $i$ th iterate, $\boldsymbol{p}$ is the model vector (i.e. the model parameters), $\boldsymbol{G}(\boldsymbol{p})$ is the forward nonlinear function used to calculate the predicted data values $\boldsymbol{d}$ from a model $\boldsymbol{p}, \boldsymbol{W}$ is a diagonal matrix whose elements are the weights described above, squared. $\boldsymbol{D}$ is the damping matrix whose non-zero elements are defined in Eq. (20) using the $N=156$ functions whose centres are at latitude higher than $40^{\circ}$ North. $\lambda$ is the usual damping parameter. $\boldsymbol{d}_{o b s}$ is the data vector and finally, $\boldsymbol{G}$ is the $n \times p$ matrix associated with the equations of condition $(n$ : number of data values; $p:$ number of model parameters):

$$
\boldsymbol{G}=\left[\frac{\partial \boldsymbol{G}_{i}(\boldsymbol{p})}{\partial \boldsymbol{p}_{j}}\right]_{\substack{i=1, n \\ j=1, p}}
$$

The linear system is solved using eigenvalue/eigenvector decomposition. The problem is regularised by removing the null or very small eigenvalues and their associated eigenvectors.

It is important to understand that when only the null eigenvalues and associated eigenvectors are removed, the output solution is exactly the one that would be obtained using a spherical harmonic representation of the magnetic field if the equivalent damping is used. This is because both the $(L+1)^{2}$ spherical harmonic functions of degree smaller or equal to $L$ and the $(L+1)(2 L+1)$ functions $F_{i j}^{L}(\theta, \varphi, a)$ span exactly the same space. However, in the solutions presented below, all eigenvalues smaller than $10^{-8}$ times the largest eigenvalue were removed for the inversion of the normal equation matrix.

Two sets of model parameters were estimated in four iterations following the process (23) and their Gauss coefficients were calculated using Eq. (11). The first set (model_1) was obtained without damping (i.e. $\lambda=0$ ) and the second set (model_2) was estimated with slight damping: $\lambda=5 \times 10^{-6}$. Table 1 gives the number of data, mean and the rms misfit for both models. The increase in the rms misfit with the damping mainly affects, as expected, the high latitude scalar data.

Figure 2 shows the power spectrum of the two models as well as the power spectrum of their differences with respect to the underlying model used to generate the synthetic data set. The sharp drop of the model's power at degree 19 is the result of the regularization process described above. It is interesting to note that the damping increases the power in degrees 14 and above. This is in contrast to a damping applied at all latitudes and longitudes and is due to an increase in the amplitude of the zonal Gauss coefficients. Indeed a combination of zonal harmonics can generate an extremely smooth magnetic field at high latitudes.

Figure 3 presents the contoured differences, for each magnetic field component, between model_1 and the reference model and also between model_2 and model_1. In the comparison of model_1 with the reference, the largest differences are mainly at high latitudes in the northern hemisphere where the noise level in the data is large. Large differences are also seen in the middle of the Atlantic Ocean. The effect of the damping can be seen in the differences 


\section{Model_1-Reference}
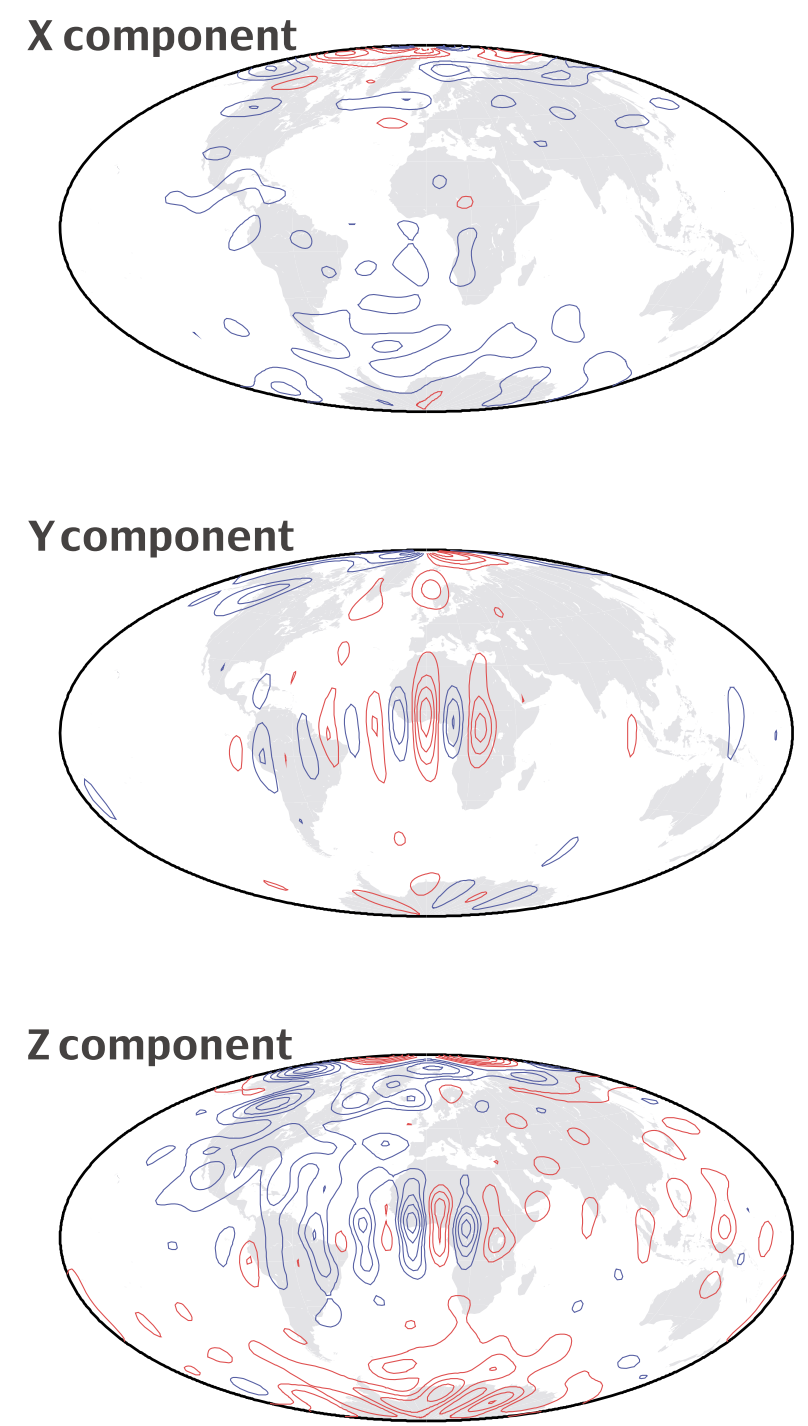

Model_2-Model_1
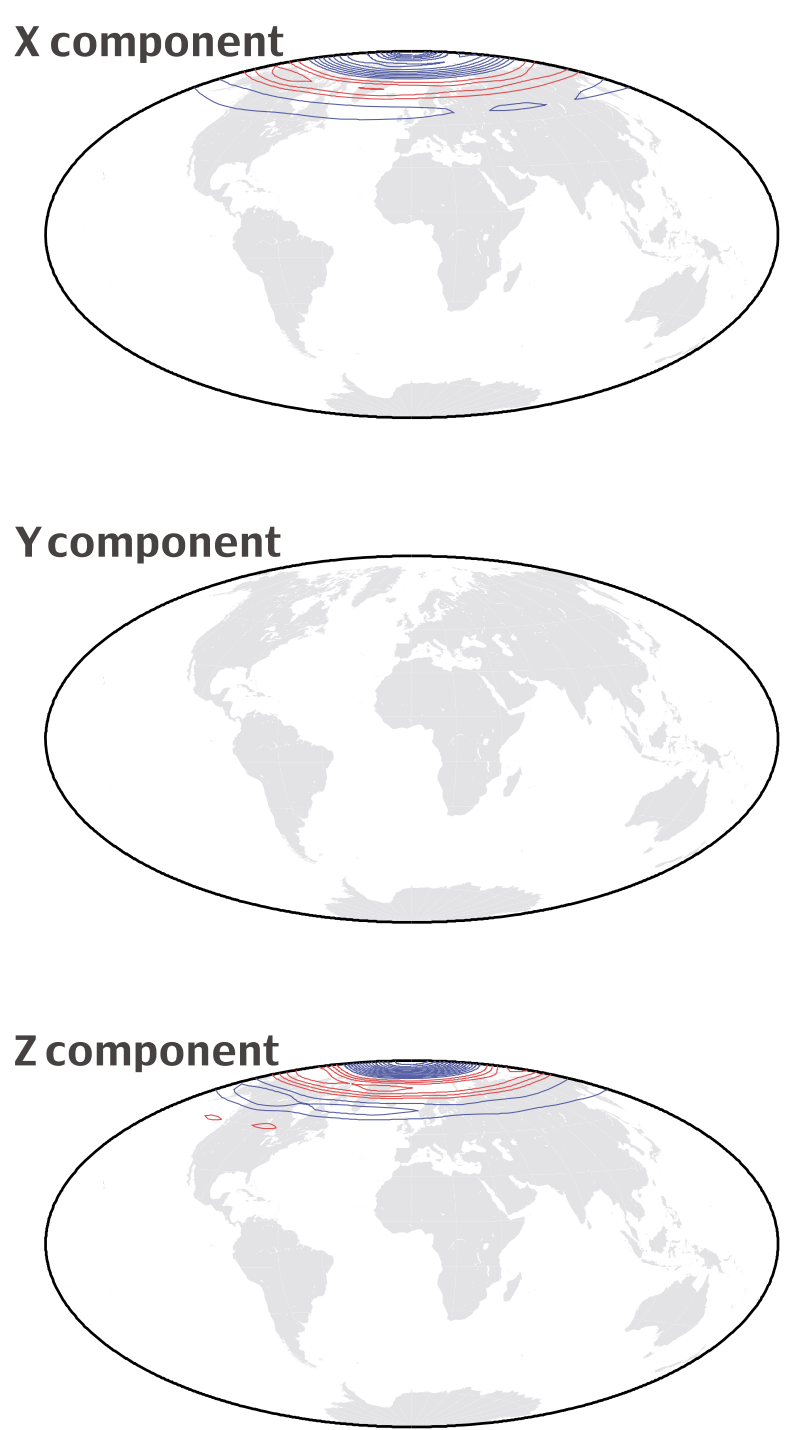

Fig. 3. Contours of the differences between, to the left, model_1 and the reference field (contour interval 10 nT, blue contours are for negative values and red positives), and to the right, model_2 and model_1 (contour interval $5 \mathrm{nT}$, blue contours are for negative values and red for positives).

between model_2 and model_1 where the contour interval is now $5 \mathrm{nT}$. These differences are large exclusively over the area where the damping was applied. Residual small differences, less than $5 \mathrm{nT}$, are present over the rest of the spherical surface in all components. It can be seen that the damping affects mainly the zonal Gauss coefficients, which was to be expected with the simple geometry and location of the damping area.

\section{Conclusion}

We have presented a technique for modelling the internal part of the geomagnetic field based on "quasi-local" functions. These functions are band-limited (i.e. they are built from a set of spherical harmonics with a given maximum degree $L$ ) which provides better control of the downward continuation of satellite data. The locations of these functions are set such that any harmonic functions of maximum degree $L$ can be represented with these "quasi-local" functions. The consequence is that any spherical harmonic model of the internal magnetic field can be easily transformed into a representation using the quasi-local functions, and conversely. The simplicity of these two transformations is the main advantage of this technique over an approach using "non-band-limited" wavelets or a truly local representation such as spherical cap harmonics.

These functions share the flexibility of other types of wavelets and should be well suited for building global or regional models of the crustal magnetic field in the presence of regional noise or un-modelled signal. However, in this work these functions were used specifically to introduce a localized constraint in a global magnetic field modelling process and we have shown how this local constraint can be applied to global spherical harmonic modelling. The technique was successfully tested on a data set built for the Swarm end-to-end simulator study.

Acknowledgments. Alan Thomson and Susan Macmillan from BGS are thanked for their help. This paper is published with 
the permission of the Executive Director of the British Geological Survey (NERC).

\section{References}

Backus, G. and J. F. Gilbert, The resolving power of gross Earth data, Geophysical Journal of the Royal Astronomical Society, 16, 169-205, 1968.

Backus, G., R. Parker, and C. Constable, Foundations of Geomagnetism, Cambridge University Press, New York, 1996.

Cui, J. and W. Freeden, Equidistribution on the sphere, SIAM J. Sci. Comput., 18(2), 595-609, 1997.

Driscoll, J. R. and D. M. Healy, Computing Fourier transforms and convolutions on the 2-sphere, Adv. Appl. Maths, 15, 202-250, 1994.

Freeden, W., T. Gervens, and M. Schreiner, Constructive Approximation on the Sphere, with Applications to Geomathematics, Clarendon Press, Oxford, 1998.

Holschneider, M., A. Chambodut, and M. Mandea, From global to regional analysis of the magnetic field on the sphere using wavelet frames, Physics of the Earth and Planetary Interiors, 135, 107-124, 2003.

Lesur, V. and D. Gubbins, Evaluation of fast spherical transforms for geophysical applications, Geophysical Journal International, 139, 547555, 1999.

Maier, T. and C. Mayer, Multiscale downward continuation of CHAMP FGM-data for crustal field modelling. First CHAMP Mission Results for Graviy, Magnetic and Atmospheric Studies, edited by Reigber, Lühr, and Schwintzer, Springer Verlag, ISBN 3-450-00206-5, pp. 288-295, 2003.

Olsen, N., R. Haagmans, T. J. Sabaka, A. Kuvshinov, S. Maus, M. E. Purucker, M. Rother, V. Lesur, and M. Mandea, The Swarm End-to-End mission simulator study: A demonstration of separating the various contributions to Earth's magnetic field using synthetic data, Earth Planets and Space, 58, this issue, 359-370, 2006.

Sabaka, T. J., N. Olsen, and M. I. Purucker, Extending comprehensive models of the Earth's magnetic field with Ørtsed and CHAMP data, Geophys. J. Int., 159, 521-547, 2004.

Sneeuw, N., Global spherical harmonic analysis by least squares and numerical quadrature methods in historical perspective, Geophysical Journal International, 118, 707-716, 1994.

V. Lesur (e-mail: v.lesur@bgs.ac.uk) 